Research Article

\title{
Dynamic Topology Optimization of Long-Span Continuum Structures
}

\author{
Yingjia Wang $\mathbb{D}^{1},{ }^{1}$ Dongchen Qin, ${ }^{2}$ Ranran Wang, ${ }^{2}$ and Heming Zhao ${ }^{2}$ \\ ${ }^{1}$ School of Mechanical Engineering, North China University of Water Resources and Electric Power, Zhengzhou 450045, China \\ ${ }^{2}$ School of Chemical Engineering and Energy, Zhengzhou University, Zhengzhou 450001, China \\ Correspondence should be addressed to Yingiia Wang; wangyingiia@ncwu.edu.cn
}

Received 1 June 2021; Accepted 31 July 2021; Published 6 August 2021

Academic Editor: Jie Yang

Copyright ( 2021 Yingjia Wang et al. This is an open access article distributed under the Creative Commons Attribution License, which permits unrestricted use, distribution, and reproduction in any medium, provided the original work is properly cited.

Herein, to improve the dynamic performance of continuum structures, their fundamental frequency is optimized using the topology optimization method. This helps to obtain the best material distribution in the design space and increases the fundamental frequency of the structure higher than the disturbance frequency. Using the variable density method, the dynamic topology optimization model of a long-span continuum structure is built based on the density interpolation model of a solid isotropic material with penalization (SIMP). The goal of this optimization is to maximize the first-order eigenvalue, and the optimization constraint is that the total volume of the structure is smaller than the given value. To improve the efficiency and accuracy of the model, sensitivity filtering is adopted to avoid numerical instability during calculation. Moreover, the optimization criterion method is used to iteratively solve the optimization results. Finally, the structural topology optimization method is implemented on the long-span single beam of a bridge crane at a construction site. The results show that the natural frequency of the structure is increased and the modal characteristics are improved, which lays the foundation for further optimization and dynamic-response analysis.

\section{Introduction}

The main concept underlying topology optimization is to maximize the material utilization rate and optimize the structural performance while satisfying certain constraints. Structural topology optimization can be traced back to the truss structure layout optimization theory proposed by Michell [1] at the beginning of the last century. In the 1980s, Bendsoe and Kikuchi [2] proposed the homogenization method, which marked the advent of research on the topology optimization of continuum structures. In recent years, topology optimization theory has developed rapidly, becoming one of the most popular and challenging fields of research in structural optimization. In actual engineering structures, particularly, long-span continuous structureswhich include cranes, hydraulic press towers, and beam structures-strict requirements must be met in terms of dynamic performance. Therefore, research on topology optimization must be extended from static design to dynamic design [3-5], and it is of great practical significance to implement dynamic topology optimization of long-span continuum structures.

When analyzing the dynamic characteristics of engineering structures, to prevent resonance and damage, the natural frequency of the structure should differ from the external excitation frequency. Moreover, it is important to study the response of a structure to various dynamic loads. Therefore, a modal analysis of the structure must be carried out first. The dynamic topology optimization of frequency focuses on improving the modal characteristics of a structure while satisfying the constraints regarding structural stiffness, such that the overall stiffness of the structure can be improved and the material can be optimized. Current topology optimization studies in the field of dynamics mainly focus on two facets of research. One is using the characteristic frequency as the objective function or constraint condition, and the other is using the structural flexibility as the objective function [6]. Li et al. [7] set up an optimization model with 
the characteristic frequency as a dynamic constraint. In this model, the dynamic constraints change according to the principal structural vibrations. Diaaz and Kikuchi [8] used the homogenization method to optimize the structural topology by maximizing the natural frequency as the objective function. Pedersen [9] used the variable density method to optimize the structural topology by maximizing the first eigenfrequency as the objective function. Based on the SIMP interpolation model, Qin et al. [10] established a minimum weight model using the displacement and fundamental frequency as a joint constraint, improving the dynamic performance of the structure. Xu and $\mathrm{Ma}$ [6] established a topology optimization model under frequency-excitation loads with the objective function of minimizing the dynamic flexibility. With the aim of minimizing the dynamic compliance of the structure, Jiang et al. [11] combined the equivalent static loads' method with the bidirectional evolutionary structural optimization method to optimize the continuum structural topology. Liu et al. [12] took the displacement response amplitude at a specified position of the structure in the steady state as the objective function and the structural volume as the constraint to investigate the topology optimization of a structure under harmonic-force excitation.

In comparison to static topology optimization, research on the dynamic topology optimization of engineering structures is still limited owing to the difficulty in setting up a model for continuum topology optimization, along with the large computational cost of using numerical algorithms in engineering applications. Yang et al. [13] proposed a structural topology optimization method with regular geometric constraints in combination with a method based on the changes in material properties and the bidirectional evolutionary structural optimization method, aimed at solving the structural optimization problem in the design of fuselage flutter models, which takes the modal values as the goal. Using the body's modal frequency and the vibration intensity of the engine block as the optimization objectives and the modal frequency as the constraint, Du et al. [14] established a structural vibration intensity optimization model. Taking the virtual prototype of a four-cylinder engine block as the design object, the variable density method was adopted to realize low-vibration optimization design. Jiao et al. [15] divided the optimization domain of a bridge crane girder into several subdomains. Then, the relationship between the subdomains and the optimization domain was constructed to establish a mathematical model for the periodic topology optimization of the girder. In this optimization problem, the relative density of the elements in the optimization domain was taken as the design variable and the minimum compliance under the volume constraint as the objective function. Jang et al. [16] designed a lightweight structure for a mobile harbor crane by considering the deadweight, inertial load, and wind force. Through the integrated design process of topology and shape optimization, the conceptual and basic designs of the $\mathrm{MH}$ crane were successfully obtained. Using the cross-section shape as the design variable, Kim et al. [17] conducted the topology optimization and shape optimization of a crane boom, with the minimum mass as the design objective and the static strength and dynamic stiffness of the system as the constraints.

In this paper, a topology optimization model is established by combining the topology optimization method and finite element theory. In this model, maximizing the firstorder eigenvalue is the goal, the total volume of the structure is the constraint, and the relative density of the elements is the design variable. To avoid a checkerboard lattice and the mesh-dependence phenomenon, the solid isotropic material with the penalization (SIMP) model and the optimization criterion (OC) method are used. Finally, the topology optimization calculation of a large-span continuum structure of construction machinery is implemented to improve the natural frequency and modal characteristics, which lays the foundation for further optimization design and dynamic-response analysis. The innovation of this paper is the presentation of an optimization criterion method by constructing the Lagrange function by introducing Lagrange multiplier based on Kuhn-Tucker condition to overcome the large computational cost and cope with the implicit nonlinear objective function in topology optimization of large-span engineering structures.

\section{SIMP Method in Variable Density Theory}

The material interpolation model of variable density theory converts the discrete optimization problem into a continuous optimization problem by introducing intermediate density elements [18]. Then, a penalty factor is introduced to punish the intermediate density so that the material density tends to discrete 0 and 1 , which denote the material elimination and material retention, respectively.

The most widely used interpolation models in continuum topology optimization are SIMP [19] model and RAMP [20] (rational approximation of material properties) model. The algorithm of the SIMP model has fast convergence and simple sensitivity, which can be expressed as

$$
E\left(x_{i}\right)=E_{\min }+x_{i}^{p}\left(E_{0}-E_{\min }\right), \quad i=1,2, \ldots, n,
$$

where $x_{\mathrm{i}}$ is the relative density of the $i$ th element, $n$ is the total number of discrete elements, $p$ is the penalty factor, $E\left(x_{\mathrm{i}}\right)$ is the elastic modulus after interpolation, and $E_{0}$ and $E_{\text {min }}$ are the elastic modulus of the solid and eliminated material, respectively.

Equation (1) cannot be directly applied to the frequency optimization for dynamic characteristics of the structure. The reason is that the penalty factor only penalizes the stiffness in low-density region, which results in the local modal with large mass and small stiffness. To avoid this phenomenon, the modified SIMP interpolation model was introduced to weigh the ratio of mass to stiffness [21], which can be written as

$$
E\left(x_{i}\right)=E_{0}\left[\frac{x_{\min }-x_{\min }^{p}}{1-x_{\min }^{p}}\left(1-x_{i}^{p}\right)+x_{i}^{p}\right], \quad 0<x_{\min } \leq x_{i} \leq 1,
$$


where $x_{\min }$ is the minimum density to avoid the singularity introduction of the stiffness matrix.

\section{Dynamics Topology Optimization Model Subheadings}

3.1. Optimization Model. Tens of thousands of points will be produced in long-span continuum structure when the finite element meshing is carried out. Therefore, a great amount of computational cost is involved to calculate the natural frequency and mode shape of each order of the structure. Moreover, the low-order mode has a stronger influence on the dynamic characteristics of the structure than the highorder mode [22]. Therefore, a dynamic optimization model with maximization of first-order eigenvalue as the goal, total volume of the structure as the constraint, and relative density of elements as the design variable is established in this paper, which is expressed as follows [23]:

$$
\begin{aligned}
& \text { Find }: \mathbf{x}=\left\{\mathbf{x}_{1}, \mathbf{x}_{2}, \ldots, \mathbf{x}_{\mathbf{n}}\right\}^{\mathbf{T}} \text {, } \\
& \text { Maximum : } \boldsymbol{\lambda}_{\min }=\min \left(\boldsymbol{\lambda}_{1}, \boldsymbol{\lambda}_{2}, \ldots, \boldsymbol{\lambda}_{\mathrm{N}_{\mathrm{dof}}}\right) \text {, } \\
& \sum_{i=1}^{n} v_{i} x_{i}-f v_{0} \leq 0, \quad i=1,2, \ldots, n, \\
& \text { Subject to : }\left(\mathbf{K}-\lambda_{j} \mathbf{M}\right) \phi=0, \quad j=1,2, \ldots, N_{d o f} \text {, } \\
& 0<x_{\min } \leq x i \leq 1 \text {, }
\end{aligned}
$$

where $\lambda_{\mathrm{j}}$ is the $j$ th eigenvalue, $N_{\mathrm{dof}}$ is the number of freedom degrees of the structure, $\phi=\left\{\phi_{1}, \phi_{2}, \ldots, \phi_{j}\right\}, \phi_{j}$ is the corresponding characteristic mode, $\mathbf{K}$ and $\mathbf{M}$ are the global stiffness matrix and mass matrix of the structure, respectively, $v_{i}$ is the $i$ th volume, $f$ is the percentage of the retained volume, which is taken as 0.5 in this paper, and $v_{0}$ is the original volume of the structure, $\rho_{0}$ is the density of solid material, and $j=1,2, \ldots, N_{\text {dof }}$ is the number of all eigenvalues corresponding to the degree of freedom of the structure.

3.2. Sensitivity Analysis. Sensitivity analysis model characteristics are sensitive to design variables. Through sensitivity analysis, design variables that have a greater impact on structural characteristics can be identified to find the optimal direction for each iteration.

Sensitivity of objective function to design variables is

$$
\phi^{T} \frac{\partial \mathbf{K}}{\partial x_{i}} \phi-\frac{\partial \lambda_{j}}{\partial x_{i}} \phi^{T} \mathbf{M} \phi-\lambda_{j} \phi^{T} \frac{\partial \mathbf{M}}{\partial x_{i}} \phi=0 .
$$

After derivation, the following equations can be obtained:

$$
\frac{\partial \lambda_{j}}{\partial x_{i}}=\phi^{T}\left(\frac{\partial \mathbf{K}}{\partial x_{i}}-\lambda_{j} \frac{\partial \mathbf{M}}{\partial x_{i}}\right) \phi
$$

Substituting (2) into equations (4) and (5), we can be obtain

$$
\frac{\partial \lambda_{j}}{\partial x_{i}}=\phi^{\mathrm{T}}\left(\sum_{i=1}^{n} \frac{1-x_{\min }}{1-x_{\min }^{p}} p x_{i}^{p-1} \mathbf{k}_{i}^{0}-\lambda_{j} \sum_{i=1}^{n} \mathbf{m}_{i}^{0}\right) \phi,
$$

where $\mathbf{k}_{i}^{0}$ is the initial stiffness of the element and $\mathbf{m}_{i}^{0}$ is the initial mass of the element.

Sensitivity of the volume constraint relative to the design variable is as follows:

$$
\frac{\partial\left(\sum_{i=1}^{n} v_{i} x_{i}-f v_{0}\right)}{\partial x_{i}}=v_{i}
$$

3.3. Optimization Criterion Method. With large-scale discrete elements and design variables, topology optimization of long-span continuum structures is very complex. To overcome the large computational cost and cope with the implicit nonlinear objective function, it is a good choice to adopt the optimization criterion method which has the obvious advantages of less iteration times and fast convergence. Based on Kuhn-Tucker condition, the optimization criterion method is to construct Lagrange function by introducing Lagrange multiplier. It requires that the number of reanalysis will not change with the complexity of structure and the number of design variables. The constrained extreme value problem is more suitable for solving the continuum topology optimization problem.

According to the Kuhn-Tucker condition, if the constraint is a nonactive constraint, then there exists a nonzero and nonnegative Lagrange multiplier $l$ if $\left(\partial c(\mathbf{X}) / \partial x_{i}\right)+l\left(\partial v(\mathbf{X}) / \partial x_{i}\right)=0$, and the solution reaches the extreme value.

The Lagrange function of the dynamic topology optimization problem is as follows:

$$
\begin{aligned}
L= & \lambda_{\min }+l_{1}\left(\sum_{i=1}^{n} v_{i} x_{i}-f v_{0}\right)+l_{2}\left[\left(\mathbf{K}-\lambda_{j} \mathbf{M}\right) \phi\right] \\
& +l_{3} \sum_{i=1}^{n}\left(x_{\min }-x_{i}\right)+l_{4} \sum_{i=1}^{n}\left(x_{i}-x_{\max }\right),
\end{aligned}
$$

where $l_{1}, l_{2}, l_{3}$, and $l_{4}$ are Lagrange multipliers.

When the design variables are the extreme value, the Kuhn-Tucker condition can be written as

$$
\begin{aligned}
& \left\{\phi_{\min }\right\}^{T}\left(\frac{\partial \mathbf{K}}{\partial x_{i}}-\lambda_{\min } \frac{\partial \mathbf{M}}{\partial x_{i}}\right)\left\{\phi_{\min }\right\} \\
& \quad+l_{2}\left(\frac{\partial \mathbf{K}}{\partial x_{i}}-\frac{\partial \lambda_{j}}{\partial x_{i}} \mathbf{M}-\lambda_{j} \frac{\partial \mathbf{M}}{\partial x_{i}}\right) \phi+l_{1} v_{i}=0 .
\end{aligned}
$$

The following formula is used for iterative update of design variables: 


$$
x_{i}^{*}= \begin{cases}\max \left(x_{\min }, x_{i}-t\right), & \text { if } x_{i} B_{i}^{\eta} \leq \max \left(x_{\min }, x_{i}-t\right), \\ \min \left(1, x_{i}+t\right), & \text { if } x_{i} B_{i}^{\eta} \geq \min \left(1, x_{i}+t\right), \\ \left(x_{i} B_{i}^{\eta}\right)^{q}, & \text { if } \max \left(x_{\min }, x_{i}-t\right)<x_{i} B_{i}^{\eta}<\min \left(1, x_{i}+t\right),\end{cases}
$$

where

$$
B_{i}=\frac{\max \left(0,-\left(\partial \lambda_{\min } / \partial x_{i}\right)\right)}{l_{1} v_{i}} .
$$

In equation (10), $x_{i}^{*}$ is the iterative design variable, $\eta$ is the damping coefficient, and $t$ represents the limit constant of movement. In this paper, $\eta=0.5$ and $t=0.2$ are used to improve the convergence of the algorithm and ensure the stability of iteration. The Lagrange multiplier $l_{1}$ is obtained by dichotomy, and $q=1$ indicates that this is the standard optimization criterion method.

\section{Filtering Function}

The optimization calculation in this paper is conducted based on the finite element method, and numerical instability will occur during the optimization process, such as checkerboard pattern and mesh dependence [24]. These phenomena make the optimization results unavailable in actual engineering structures. In order to suppress this problem, it is necessary to introduce the filtering function in the postprocessing. The commonly used filtering methods include density filtering, sensitivity filtering, and gray filtering [25]. In this paper, the sensitivity filtering method is adopted; its basic idea is to use the weighted average sensitivity value to replace the old sensitivity to achieve numerically unstable filtering.

The sensitivity filtering method proposed by Sigmund [26] has a good effect in overcoming numerical instability. This method replaces the original sensitivity value of the central element with the weighted average of the sensitivity of the element within the filtering radius. The classical sensitivity filtering formula is

$$
\begin{aligned}
\frac{\partial \widehat{c}}{\partial x_{i}} & =\left(\frac{1}{x_{i} \sum_{k=1}^{N} H_{k}}\right) \sum_{k=1}^{N} H_{k} x_{k} \frac{\partial c}{\partial x_{k}}, \\
H_{k} & =r-r_{1}(i, k),
\end{aligned}
$$

where $r$ is the filtering radius, $c$ is the objective function, $N$ is the total number of all elements in the range from the center element, $H_{k}$ is the convolution factor, and $r_{1}(i, k)$ is the distance between the center of elements $i$ and $k$, usually taking 1-3 times of the element size.

Gray filtering [27] is a nonlinear filtering method to obtain clearer black-and-white cells, also known as intermediate density filtering method. It can be realized by modifying the parameters in OC update criteria. For example, the coefficient $q$ in equation (11) can be taken as 2 .

\section{Topology Optimization of a Large-Span Beam Structure}

5.1. Mechanical Model. Taking the single main girder of a large-span normal-track double-beam bridge crane as an example, its mechanical model can be simplified to a simply supported solid girder structure. The force analysis is carried out when the trolley is fully loaded at the midpoint of the span, and the static wheel pressure of the trolley and the weight of the beam are equivalent to the contact point of the wheel and the beam, yielding the following parameters: $P=115 \mathrm{kN}$, beam span $L=21.6 \mathrm{~m}$, height $B=1.3 \mathrm{~m}$, width $S=0.5 \mathrm{~m}$, small wheel pitch $C=3.6 \mathrm{~m}$, and $E_{0}=210 \mathrm{GPa}$. The corresponding force diagram is shown in Figure 1. The beam is fixed at two ends of lower cover plate. An eight-node regular hexahedron element with a size of $0.1 \mathrm{~m}$ size was used for meshing. The optimal area is set as the solid portion between the upper and lower cover plates. In comparison to the main girder and trolley, the weight of the driver's cab, the walkway, and the electrical system is very small and is ignored in the design process.

5.2. Flowchart of Topology Optimization. The process of girder structural dynamic topology optimization is shown in Figure 2. The precision of convergence is prescribed to be 0.01 .

5.3. Results and Analysis. It can be seen from equation (2) that intermediate densities incur a penalty. However, too large a penalty factor makes elements with relative densities approaching 1 tend to 0 , and too small a penalty factor nullifies the effect of using penalties. Therefore, the selection of the penalty factor affects the optimized structure. Figure 3 shows the topology optimization results when $p=3,4,5$, and 7 and $r=1.5 \mathrm{~m}$. Figure 4 shows the frontal view of the optimization results with different penalty factors. It can be seen that there is a hollow structure inside the beam, which is consistent with the general box-type structure of main beams of cranes in practice. Moreover, holes gradually appear in the web. With the increases in the penalty factor, the number, shape, and location of holes are different, and the retained material generally presents an inclined distribution. These results are consistent with [18].

The material on the web has an inclined distribution, the web material in the middle part is removed, and the trapezoidal structure is used to bear the pressure of the two wheels of the trolley. The structure on the web is generally inclined, indicating that the web material is best arranged in this way under these working conditions. Therefore, the 


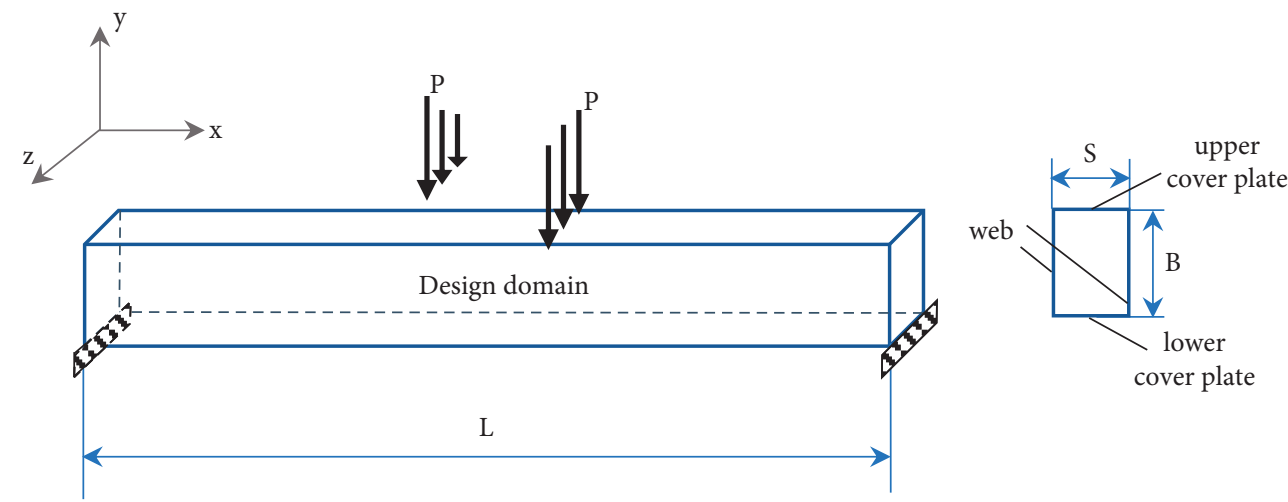

Figure 1: Force diagram of the single girder.

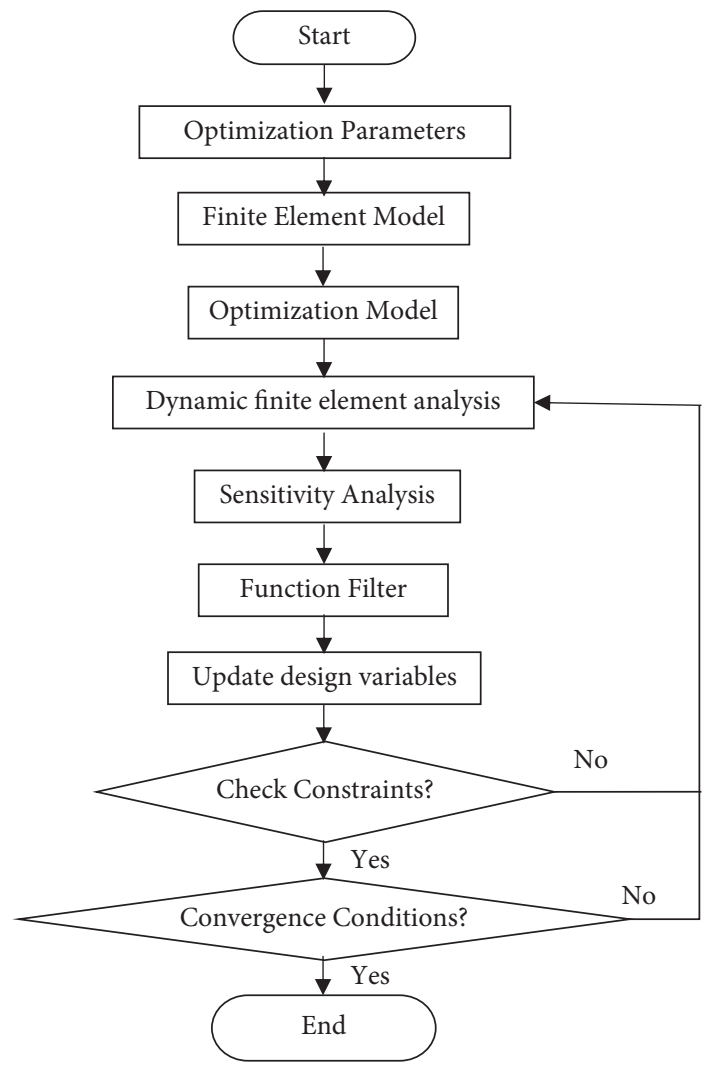

FIGURE 2: Flowchart of the proposed topology optimization method.

inclined layout of the internal ribs of the web can be considered in actual practice. When $p=1$, there is no penalty effect and an unreasonable structure is obtained through optimization. When $p=3$, a continuous inclined structure appeared on the web, indicating that the penalty factor began to take effect, and the thickness of the longest inclined structure was relatively thin. When $p=4-5$, the penalty effect is more obvious. In comparison to $p=3$, the inclined structure on the web is reduced, but the longest part is thickened. When $p=7-9$, the penalty effect is too heavy; too much material is removed and the structure tends to become truss-like.

Figure 5 shows the fundamental frequency optimization process with different penalty factors when $r=1.5 \mathrm{~m}$. The top figure shows the complete iterative process, while the bottom figure shows a partially enlarged view of the first 40 iterations. With increases in the iteration number, the first-order natural frequency of the structure first decreases slightly and then increases rapidly, finally slowly increasing after 30 iterations until the end of the iteration process. Moreover, with increases in the penalty factor, the number of iterations increases, and the frequency optimization result gradually decreases.

The track direction of the bridge crane trolley reflects the first-order natural frequency of transverse horizontal vibrations. According to the corresponding design requirements in China, the frequency along the direction of the bridge crane on the trolley track should be greater than $1 \mathrm{~Hz}$. As shown in Table 1 -other than the fact that $p=1$ does not reflect any effects of penalties-the fundamental frequency values before optimization are less than $1 \mathrm{~Hz}$. When using other values for $p$, the optimal structure has different fundamental frequency values, all of which are greater than $1 \mathrm{~Hz}$, which meet the requirements for dynamic design.

Figure 6 shows the optimization process of the fundamental frequency under different filtering radii at $p=3$. The figure at the top shows the full iteration process, while the figure at the bottom shows a partially enlarged view of the first 40 iterations. As the number of iterations increases, the first-order natural frequency of the structure also increases. When $r=1.0-1.2 \mathrm{~m}$, the frequency value increases slightly, and as the filter radius increases, the frequency optimization result gradually decreases. The fundamental frequency values of the structure after optimization from Table 1 are all greater than $1 \mathrm{~Hz}$, which meets the requirements of dynamic characteristic design.

Figure 7 shows the front view of structural topology optimization results with different filter radii. When $r=1 \mathrm{~m}$, it can be seen that a large amount of material is retained on the web, and there is an obvious checkerboard phenomenon. When $r=1.2 \mathrm{~m}$, there are too many fine structures on the web, but the retained material has a clear inclined arrangement. When $r=1.5 \mathrm{~m}$, there are no unnecessary details in the structure, and the material distribution is more reasonable. When $r=1.8-2.5 \mathrm{~m}$, the web structure is removed excessively, and some structural features are deleted. 


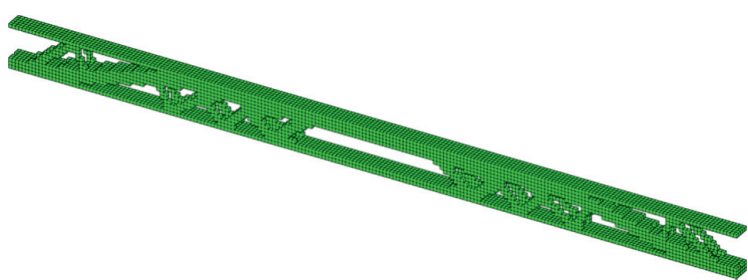

(a)

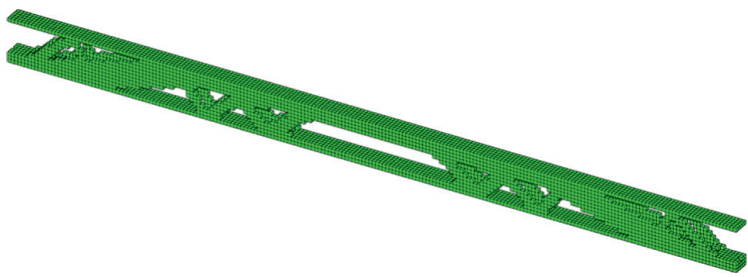

(c)

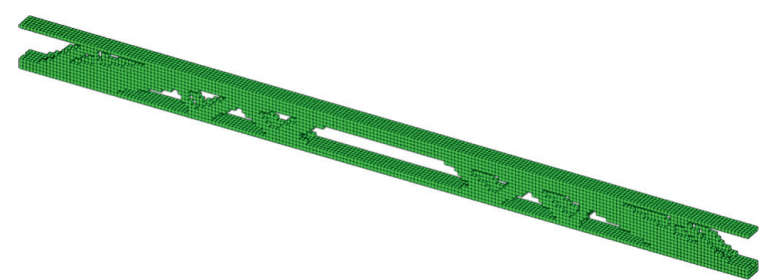

(b)

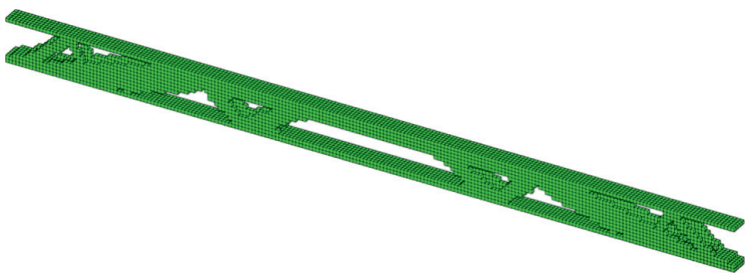

(d)

Figure 3: The result of structural topology optimization $(r=1.5 \mathrm{~m}$ ). (a) $p=3$. (b) $p=4$. (c) $p=5$. (d) $p=7$.

(a)

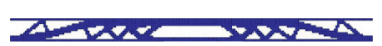

(b)

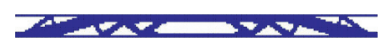

(c)

(d)

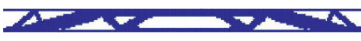

(e)

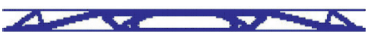

(f)

Figure 4: Front view of structural topology optimization results with different penalty factors $(r=1.5 \mathrm{~m})$. (a) $p=1$. (b) $p=3$. (c) $p=4$. (d) $p=5$. (e) $p=7$. (f) $p=9$.

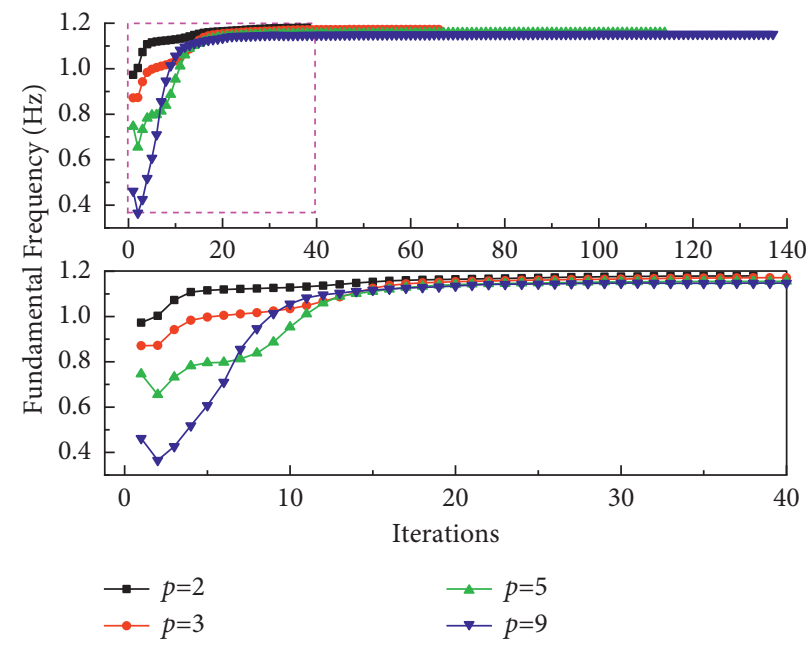

FIGURE 5: Optimization process of the fundamental frequency with different penalty factors $(r=1.5 \mathrm{~m})$.

TABLe 1: Fundamental frequency values under different penalty factors and filter radii.

\begin{tabular}{lcccccccccc}
\hline & \multicolumn{3}{c}{$r=1.5 \mathrm{~m}$} & \multicolumn{3}{c}{$p=3$} \\
& $p=2$ & $p=3$ & $p=5$ & $p=7$ & $p=9$ & $r=1 \mathrm{~m}$ & $r=1.2 \mathrm{~m}$ & $r=1.5 \mathrm{~m} \quad r=1.8 \mathrm{~m} \quad r=2 \mathrm{~m}$ \\
\hline Before optimization & 0.973 & 0.871 & 0.746 & 0.615 & 0.462 & 0.871 & 0.871 & 0.871 & 0.871 \\
The optimized & 1.180 & 1.172 & 1.159 & 1.145 & 1.144 & 1.180 & 1.192 & 1.172 & 1.159 & 1.150 \\
\hline
\end{tabular}

Figure 8 shows the volume ratio $\left(v_{i} / v_{0}\right)$ curves in the iterative process with different penalty factors when $r=1.5 \mathrm{~m}$. Figure 9 shows the volume ratio $\left(v_{i} / v_{0}\right)$ curves in the iterative process with a different filter radius when $p=3$. The volume ratio increases slowly and then increases rapidly after about 30 iterations and finally converges to 0.5 after 


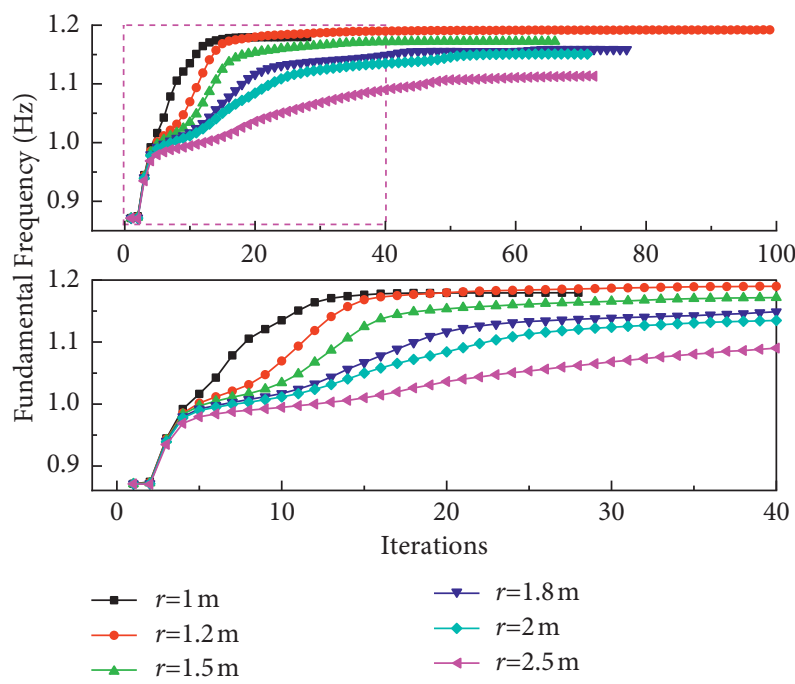

Figure 6: Optimization process of the fundamental frequency at different filter radii $(p=3)$.

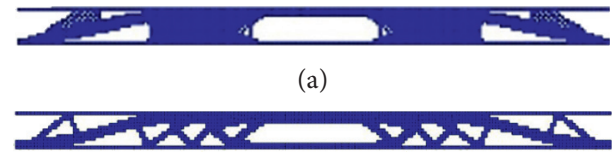

(c)

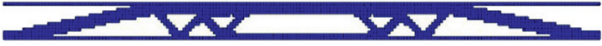

(e)

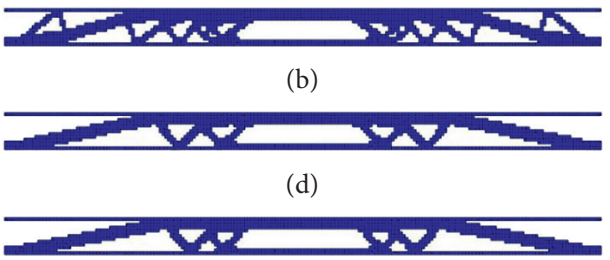

(f)

FiguRe 7: Front view of structural topology optimization results with different filter radius ( $p=3$ ). (a) $r=1 \mathrm{~m}$. (b) $r=1.2 \mathrm{~m}$. (c) $r=1.5 \mathrm{~m}$. (d) $r=1.8 \mathrm{~m}$. (e) $r=2 \mathrm{~m}$. (f) $r=2.5 \mathrm{~m}$.

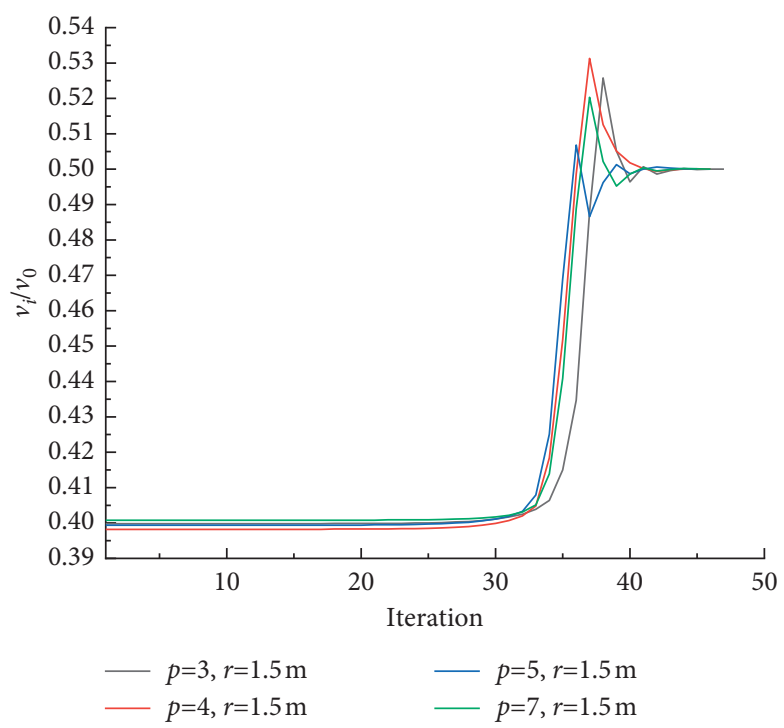

Figure 8: Volume ratio curves in the iterative process with different penalty factors $(r=1.5 \mathrm{~m})$. 


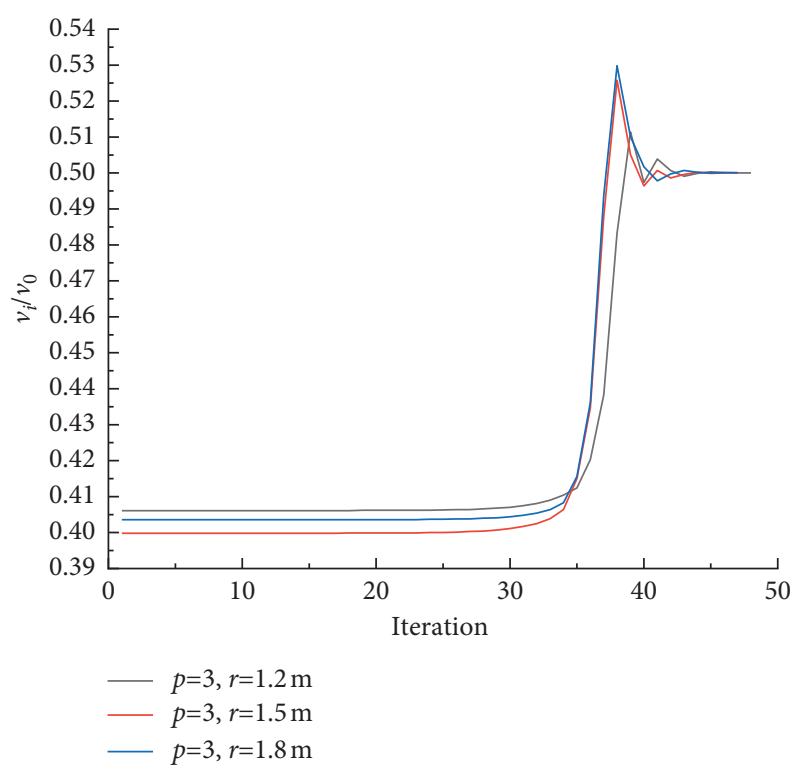

FIGURE 9: Volume curves in the iterative process with different filter radii $(p=3)$.

several oscillations. The curves and those in [18] have the similar variation tendency.

\section{Conclusions}

In construction machinery, large-span continuum structures have a large number of model elements and design variables. Therefore, a large computational cost is involved in their performance analysis and design optimization. In this article, the dynamic characteristics of the aforementioned structure are optimized based on the variable density method. The optimization model is established with the goal of maximizing first-order eigenvalues with a constraint regarding the total volume. Sensitivity filtering is used to suppress numerical instability while improving the efficiency and accuracy of calculations. The optimization criterion method is used to efficiently solve the structure and obtain the corresponding topological structure to increase its fundamental frequency. The proposed method can meet the requirements of dynamic characteristics design and improve the utilization rate of materials. Therefore, the obtained topological structure can be used as a reference to guide future studies on design.

\section{Data Availability}

The data used to support the findings of this study are included within the article. The topology optimization results in this paper have been obtained using the software Matlab. The codes have been uploaded as the supplementary material.

\section{Conflicts of Interest}

The authors declare no conflicts of interest.

\section{Acknowledgments}

The authors are grateful for financial support from projects in the National Science and Technology Pillar Program (Grant no. 2015BAF06B06).

\section{Supplementary Materials}

MATLAB codes of the proposed topology optimization method. (Supplementary Materials)

\section{References}

[1] A. G. M. Michell, "LVIII. The limits of economy of material in frame-structures," The London, Edinburgh, and Dublin Philosophical Magazine and Journal of Science, vol. 8, no. 47, pp. 589-597, 1904.

[2] M. P. Bendsoe and N. Kikuchi, "Generating optimal topologies in structural design using a homogenization method," Computer Methods in Applied Mechanics and Engineering, vol. 71, pp. 197-224, 1988.

[3] G. I. Rozvany, "A critical review of established methods of structural topology optimization," Structural and Multidisciplinary Optimization, vol. 37, pp. 217-237, 2019.

[4] J. D. Deaton and R. V. Grandhi, "A survey of structural and multidisciplinary continuum topology optimization: post 2000," Structural and Multidisciplinary Optimization, vol. 49, no. 1, pp. 1-38, 2014.

[5] J. Pang, W. G. Zheng, and L. Yang, "Topology optimization of free damping treatments on plates using level set method," Shock and Vibration, vol. 2020, Article ID 5084167, 17 pages, 2020.

[6] J. Q. Xu and Y. Q. Ma, "Topology optimization of continuum structures under frequency excitation load based on dynamic natural element method," Journal of Vibration and Shock, vol. 38, pp. 252-258, 2019.

[7] J. Li, S. Chen, and H. Huang, "Topology optimization of continuum structure with dynamic constraints using mode identification," Journal of Mechanical Science and Technology, vol. 29, no. 4, pp. 1407-1412, 2015.

[8] A. R. Díaaz and N. Kikuchi, "Solutions to shape and topology eigenvalue optimization problems using a homogenization method," International Journal for Numerical Methods in Engineering, vol. 35, no. 7, pp. 1487-1502, 1992.

[9] N. L. Pedersen, "Maximization of eigenvalues using topology optimization," Structural and Multidisciplinary Optimization, vol. 20 , no. 1, pp. 2-11, 2000.

[10] D. C. Qin, H. M. Zhao, and R. R. Wang, "Static-dynamic combined topology optimization of continuum structure," Machine Design and Manufacture, vol. 11, pp. 29-32, 2018, in Chinese.

[11] X. D. Jiang, Z. Liu, and X. Y. Teng, "Topological optimization of dynamic stiffness in continuum structure based on bidirectional evolutionary structural optimization," Journal of Harbin University of Science and Technology, vol. 25, pp. 136-142, 2020.

[12] H. Liu, W. H. Zhang, and J. H. Zhu, "Structural topology optimization and frequency influence analysis under harmonic force excitations," Chinese Journal of Theoretical and Applied Mechanics, vol. 45, pp. 588-597, 2013.

[13] R. Yang, Y. Liu, and W. Qian, "Topology optimization for structural design of fuselage flutter model," Journal of Mechanical Engineering, vol. 47, no. 11, pp. 59-63, 2011. 
[14] X. Du, Z. J. Bi, and F. R. Zhang, "Block design of diesel engine for low vibration level based on topology and shape optimization," Journal of Mechanical Engineering, vol. 48, no. 9, pp. 117-122, 2012.

[15] H. Jiao, Q. C. Zhou, and Q. L. Wu, "Periodic topology optimization of the box-type girder of bridge crane," Journal of Mechanical Engineering, vol. 50, no. 23, pp. 134-139, 2014.

[16] G. Jang, K.-S. Kim, and B. M. Kwak, "Conceptual and basic designs of the Mobile Harbor crane based on topology and shape optimization," Structural and Multidisciplinary Optimization, vol. 50, pp. 505-515, 2014.

[17] Y. C. Kim, J. K. Hong, and G. W. Jang, "Lightweight crane design by using topology and shape optimization," Transactions of the Korean Society of Mechanical Engineers A, vol. 7, pp. 821-826, 2011.

[18] H. Jiao, Q. C. Zhou, and W. J. Li, "Periodic topology optimization using variable density method," Journal of $\mathrm{Me}$ chanical Engineering, vol. 49, pp. 132-138, 2013.

[19] S. T. Liu and D. He, "SIMP-based evolutionary structural optimization method for topology optimization," Chinese Journal of Computational Mechanics, vol. 26, pp. 761-777, 2009.

[20] A. Garaigordobil, R. Ansola, J. Santamaría, and I. F. d. Bustos, "A new overhang constraint for topology optimization of selfsupporting structures in additive manufacturing," Structural and Multidisciplinary Optimization, vol. 58, pp. 2003-2017, 2018.

[21] X. Huang, Z. H. Zuo, and Y. M. Xie, "Evolutionary topological optimization of vibrating continuum structures for natural frequencies," Computers and Structures, vol. 88, pp. 357-364, 2009.

[22] J. Chen, Z. L. Yang, and Y. C. An, "Dynamic characteristics analysis and topology optimization for column based on ANSYS," Journal of Machine Design, vol. 32, pp. 61-65, 2015.

[23] S. Y. Li, R. R. Wang, and P. Zhou, "Design research on topology optimization of crane girder," China Heavy Equipment, vol. 1, pp. 20-23, 2020.

[24] M. Zhou, Y. K. shyy, and H. L. Thomas, "Checkerboard and minimum member size control in topology optimization," Structural and Multidisciplinary Optimization, vol. 21, pp. 152-158, 2001.

[25] Z. Shang and Y. K. Sui, "The impact of filter function on optimization efficiency in structural topology optimization," Machinery Design \& Manufacture, vol. 8, pp. 244-246, 2014.

[26] O. Sigmund, "A 99 lines topology optimization code written in Matlab," Structural and Multidisciplinary Optimization, vol. 21, pp. 120-127, 2001.

[27] A. A. Groenwold and L. F. P. Etman, "A simple heuristic for gray-scale suppression in optimality criterion-based topology optimization," Structural and Multidisciplinary Optimization, vol. 39, pp. 217-225, 2009. 